\title{
Ulcerative colitis complicating seronegative HLA-A2-B27 rheumatoid arthritis with sacroiliitis
}

\author{
T Klausen, K Amris, $\mathbf{P}$ Helin
}

\begin{abstract}
The case is reported of a 50 year old man with longstanding seronegative rheumatoid arthritis who developed ulcerative colitis. The patient also had sacroiliitis and his tissue was typed as HLA-A2-B27 several years before the bowel disease began. A possible overlap between primary inflammatory bowel disease, complications to the treatment of rheumatoid arthritis with drugs, and gastrointestinal rheumatoid vasculitis is discussed.
\end{abstract}

(Ann Rheum Dis 1992; 51: 1009-1011)

Intestinal inflammation and ulceration are recognised complications to the treatment of rheumatoid arthritis (RA) with drugs. ${ }^{1-3}$ In addition, a specific form of peripheral arthritis and sacroiliitis affects as many as $15-20 \%$ of patients with known ulcerative colitis or Crohn's disease. ${ }^{4}$ The histocompatibility antigen HLAB27 seems to be the link between inflammatory bowel disease, sacroiliitis and seronegative arthritis. Vasculitic lesions and chronic inflammation also occur in the gastrointestinal tract of patients with RA without gastrointestinal symptoms. ${ }^{5}$ This suggests an overlap between inflammatory bowel disease, HLA-B27, sacroiliitis, and (seronegative) RA. We report here a case of ulcerative colitis in a patient with

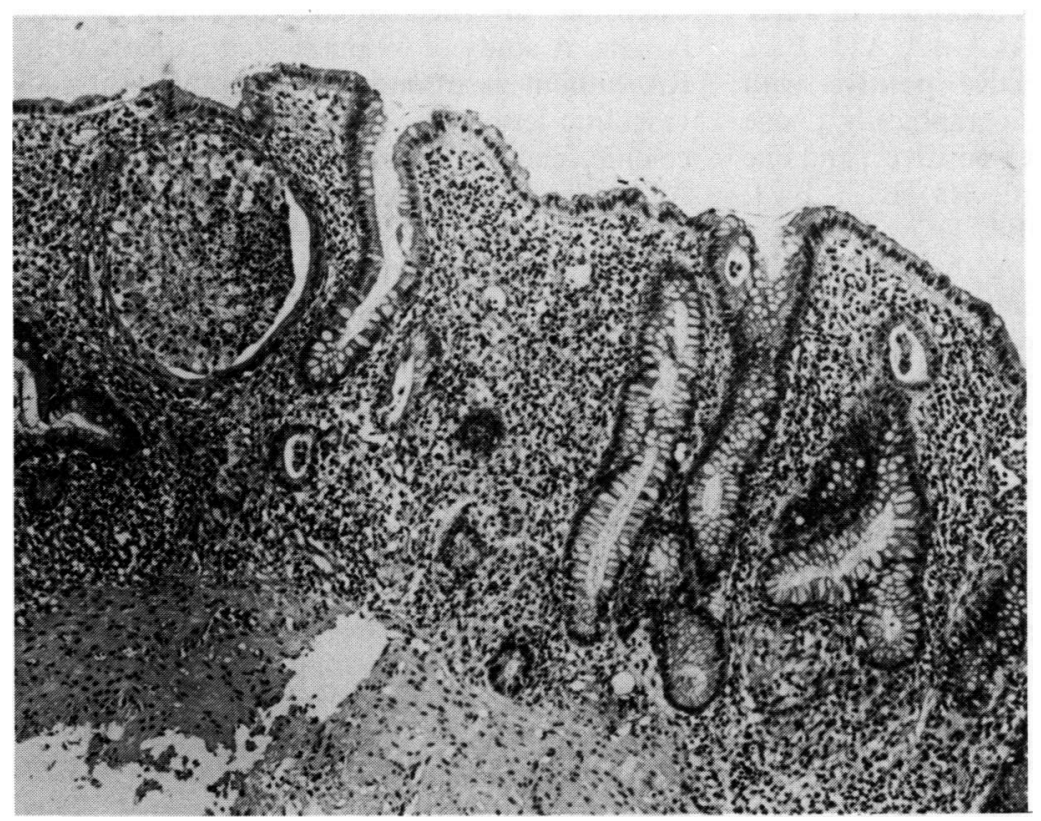

longstanding seronegative RA, tissue type HLA-B27, and sacroiliitis.

\section{Case report}

A 50 year old man had a 33 year history of RA with progressive joint destruction. The diagnosis was made according to the 1987 American Rheumatism Association criteria. ${ }^{6}$ Clinical examination revealed chronic deformity of all metacarpophalangeal and metatarsophalangeal joints and capsular thickening of the knees. Radiographs showed bilateral sacroiliitis without syndesmophytes or ankylosis of the vertebral column. The patient had a Lubinus prothesis in the right hip joint, and subchondral cysts and sclerosis of the knee joints. Serial radiographs of the hands and feet showed progressive erosions and later destructions of the carpometacarpal, metacarpophalangeal, proximal interphalangeal, and metatarsophalangeal joints. Operations were carried out on several of these joints. A radiograph with tomography of the cervical column showed erosion of dens epistrophei with atlantoaxial subluxation.

Laboratory findings included positive results for HLA-A2, A24, B27, and Cw1. There were increased gammaglobulins ( $28 \mathrm{~g} / 1$, normal range 7-13 g/l), a negative titre for antibodies to DNA, a positive titre for antinuclear antibodies, negative latex and Rose-Waaler tests, and negative IgM results for rheumatoid factor and antibodies to yersinia. In a period with low clinical disease activity the erythrocyte sedimentation rate (ESR) (Westergren) ranged from 24 to $50 \mathrm{~mm} / \mathrm{h}$, and haemoglobin concentrations were 89 to $111 \mathrm{~g} / \mathrm{l}$. The initial treatment was prednisone in variable doses by mouth (5-20 mg/day), piroxicam $20 \mathrm{mg} /$ day (later reduced to $20 \mathrm{mg}$ every second day), and indomethacin $50 \mathrm{mg}$ twice daily (later reduced to $50 \mathrm{mg} /$ day $)$. Penicillamine $(500 \mathrm{mg} /$ day $)$ wãs added after 23 years of disease, and sulphasalazine ( $500 \mathrm{mg}$ four times a day) after 29 years of disease. The patient had never received chrysotherapy. Treatment with penicillamine was complicated by ulcerations of the mouth. These were successfully treated with local steroid ointments and local excisions.

After 32 years of RA, the patient lost $10 \mathrm{~kg}$ in weight and passed 10-20 watery, blood mixed loose stools a day. Laboratory findings revealed blood glucose levels from $10-20 \mathrm{mmol} / \mathrm{l}$, an ESR increasing to $70 \mathrm{~mm} /$ hour, haemoglobin concentrations between 69 and $85 \mathrm{~g} / \mathrm{l}$, and leucocyte counts between 10 and $13 \times 10^{9} / 1$. Rectoscopy and biopsy samples showed haemo- 
rrhagic proctitis and infiltration of the mucosa and lamina propria with granulocytes, plasma cells, and lymphocytes. No granulomas, giant cells, nor amyloid deposits were seen. Colonoscopy and biopsy samples showed inflammation of the whole colon, infiltration of the mucosa and lamina propria with granulocytes, plasma cells, lymphocytes, and microabcesses (figure). No granulomas, giant cells, nor amyloid deposits were observed. A barium enema and culture of stool specimens showed no abnormalities. This was consistent with ulcerative colitis. The specific treatment given was sulphasalazine $1.5 \mathrm{~g}$ three times a day, prednisone enemas $10 \mathrm{mg} /$ day, prednisone by mouth, subcutaneous injections of insulin, blood transfusions, and intravenous hydration. Treatment with penicillamine, piroxicam and indomethacin was discontinued. Surgamyl was added. This reduced the activity of the ulcerative colitis to one to two normal stools per day, and the clinical activity of RA remained low.

\section{Discussion}

To our knowledge, no other cases of RA with concomitant HLA-A2-B27, sacroiliitis, and complicating ulcerative colitis have been reported. Intestinal damage is, however, a recognised complication of drug treatment in RA. ${ }^{1-3}$ Thirty two cases of gold induced colitis have been reported between 1929 and $1988 .^{278}$ Of these 26 patients had RA, three systemic lupus erythematosus, one primary chronic polyarthritis, and one undefined arthritis. In the group with RA, 19 patients were women and seven men. The serology was reported in 20 patients with RA and gold induced colitis; six patients were seronegative and 14 were seropositive (latex test, Rose-Waaler test, and IgM rheumatoid factor). Gold treatment was given orally and parenterally. Colitis developed in 24 of 26 patients within 10 weeks of treatment with gold or before a cumulative dose of $500 \mathrm{mg}$ was reached. HLA typing was reported in three patients: one patient was HLA-A2, A23, B27, Bw62, Cw2, Cw3, and DR4 positive with no sacroiliitis visible radiographically ${ }^{2}$; one patient was HLA-B8, DRw3 positive'; and one patient was HLA-A2, A10, B8, B12, Bw4, Bw6, Cw5, Cw7, DR3, DR4, DQw2, DQw3, and DRw53 positive. ${ }^{10} \mathrm{~A}$ hypersensitivity mechanism towards elemental gold could explain these cases of colitis. Stillman and Dubey $^{8}$ have investigated the lymphocyte response and autocytotoxicity to sodium aurothiomalate in a patient with RA and gold induced colitis. Their observations could not support hypersensitivity as a mechanism of gold induced colitis. The HLA types were not characterised. Proteinuria as an adverse effect to gold is associated with a high incidence of HLADRw3. ${ }^{11}$ We suggest that hypersensitivity to gold might occur in the gut of a small proportion of patients with RA, namely those with the antigens HLA-A2-B27. The structure of the HLA-A2 molecule contains a large groove providing a binding site for processed antigens. ${ }^{12}$ A similar structure is suggested for the HLAB27 molecule. ${ }^{13}$ Gold induced changes of mucosal permeability, microbial antigens, and toxin in the gut could thus supply the antigen binding groove of HLA-A2-B27 with arthritogenic and ulcerogenic peptides. This remains to be verified.

No chrysotherapy was used in this patient, but treatment included penicillamine and nonsteroidal anti-inflammatory drugs (NSAIDs). One case of colitis complicating treatment with penicillamine has been described previously, ${ }^{3}$ but the patient was also receiving methyldopa and indomethacin. Mouth ulcerations appeared during treatment with penicillamine in this patient. Thus penicillamine might have an effect on the gut similar to that of gold salts, with increased gut permeability and as a result HLA-A2-B27 presentation of ulcerogenic and arthritogenic peptides.

NSAIDs have an additional part to play. Small intestinal ulcerations and overgrowth of caecal type microorganisms have been observed in rats treated with NSAIDs. ${ }^{14}$ Two thirds of patients with RA receiving long term treatment with NSAIDs had small intestinal inflammation as shown by indium-leucocyte scans, increased small bowel permeability, and abnormal scans of the colon performed with the chromiumEDTA absorption test. ${ }^{15}$ Our patient continuously received various kinds of NSAIDs. This might increase gut permeability and decrease mucosal prostaglandin synthesis, allowing access to microorganisms and toxins. A cross reactivity between HLA-B27 and yersinia and klebsiella species has been described. ${ }^{16}$ This patient also had increased levels of circulating immunoglobulins. Thus a damaged gut mucosa during treatment with penicillamine and NSAIDs, cross reactivity between HLA types and microorganisms, and increased levels of circulating immunoglobulins with the formation of local immune complexes could all function together to yield bowel inflammation.

It is also possible that the inflammatory bowel disease of our patient merely represented a true extension of rheumatoid vasculitis to the bowels. A study of 59 unselected patients with RA without gastrointestinal symptoms showed vasculitic lesions in $4.3 \%$ of gastric, $15.7 \%$ of colonic, and $30.5 \%$ of rectal biopsy samples. ${ }^{5}$ All patients had received variable cycles of corticosteroids and NSAIDs. Only three patients were receiving gold. There was no correlation between histological changes and rheumatoid factor titres or the duration and severity of RA. It would be interesting to know the HLA types of these patients as they could represent a subset of patients with HLA-B27 as a genetic disposition to complicating bowel inflammation.

Clinical observations allow the differentiation between arthritis as a complication to inflammatory bowel disease and the opposite relation. The arthritis complicating primary inflammatory bowel disease is usually seronegative, non-erosive and most often affects large joints. ${ }^{4}$ When sacroiliitis is present the HLA-B27 antigen occurs with a high frequency. This patient had seronegative definite RA with joint erosions, sacroiliitis, and HLA-A2-B27 several years before the complicating ulcerative colitis. 
Based on this patient, we suggest the existence of a subset of seronegative patients with RA with the HLA-A2-B27 antigen and sacroiliitis. These patients represent an overlap between primary inflammatory bowel disease, bowel disease complicating the treatment of RA with drugs, and true extension of rheumatoid vasculitis to the gastrointestinal tract.

1 Madhok R, MacKenzie J A, Lee F D, Bruckner F E, Terry $T R$, Sturrock R D. Small bowel ulceration in patients receiving non steroidal anti-inflammatory drugs for rheumatoid arthritis. $Q \mathcal{F}$ Med 1986; 225: 53-8.

2 Langer H E, Hartmann G, Heinemann G, Richter K. Gold colitis induced by auranofin treatment of rheumatoid arthritis; case report and review of the literature. Ann Rheum Dis 1987; 46: 787-92.

3 Fortson W C, Tedesco F J. Drug induced colitis: a review. Am ₹ Gastroenterol 1984; 79: 878-83.

4 Spiro H M. Clinical gastroenterology. New York: Macmillan, 1983.

5 Marcolongo R, Bayeli P F, Montagnani M. Gastrointestinal involvement in rheumatoid arthritis: a biopsy study. f Rheumatol 1979; 6: 163-73.

.6 Arnett F C, Edworthy S M, Bloch D A, et al. The 1987 revised American Rheumatism Association criteria for classifi- cation of rheumatoid arthritis. Arthritis Rheum 1988; 31 : 315-24.

7 Jackson C W, Haboubi N Y, Whorwell P J, Schofield P F. Gold induced enterocolitis. Gut 1986; 27: 452-6.

8 Stillman A E, Dubey D P. Immune functions and goldinduced enterocolitis. Dig Dis Sci 1988; 33: 1046-7.

9 Sukenik S, Hirsch M, Yanai-Inbar I, Krawiec J, Freund B Horowitz Y. Enterocolitis complicating crysotherapy. $I s r \mathcal{F}$ Med Sci 1982; 18: 1040-3.

10 Bross-Bach U, Saal J G, Hartmann F, Muller C A, Waller $H$ D. Ulzerierende kolitis unter oraler goldtherapie be rheumatoider arthritis. $Z$ Rheumatol 1987; 46: $201-4$.

11 Hakala $M$, van Assendelft A $H$ W, Ilonen J, Jalava S, Tiilikainen A. Association of different HLA antigens with various toxic effects of gold salts in rheumatoid arthritis. Ann Rheum Dis 1986; 45: 177-82.

12 Bjorkmann P S, Saper M A, Samaroui B, Bennett W S Strominger J L, Wiley D C. Structure of the human class 1 histocompability antigen: HLA A2. Nature 1987; 329 506-12.

13 Parham P. Structure of class-1 MHC molecules: HLA B27 and disease. Scand 7 Rheumatol Suppl 1990; 87: 11-20.

14 Kent T H, Cardelli R M, Stamler F W. Small intestinal ulcers and intestinal flora in rats given indomethacin. Am $\mathcal{F}$ Pathol 1969; 54: 237-45.

15 Bjarnason I, Peters T J. Intestinal permeability, non-steroidal anti-inflammatory drug enteropathy and inflammatory bowel disease: an overview. Gut 1989; 30: 22-8.

16 Schwimmbeck P L, Yu D T Y, Oldston M B A. Autoantibodies to HLA-B27 in the sera of HLA-B27 patients with ankylosing spondylitis and Reiter's syndrome: molecular mimicry with Klebsiella pneumoniae as potential mechanisms of autoimmune disease. $\mathcal{F}$ Exp Med 1987; 166: 173-81. 